Globe

Revue internationale d'études québécoises

GLOBE

\title{
La contribution des conservateurs à la longue survie des organisations fascistes d'Adrien Arcand. Un élément d'explication
}

\section{The Contribution of the Conservatives in the Long Survival of Adrien Arcand's Fascist Organizations : an Explanatory Element. One aspect of the Conservative Party's contributions to the persistence of Adrien Arcand's fascist organizations}

\section{Jonathan Tremblay}

Volume 18, numéro 1, 2015

Nouveaux regards sur le phénomène de l'antisémitisme dans l'histoire du Québec

URI : https://id.erudit.org/iderudit/1037877ar

DOI : https://doi.org/10.7202/1037877ar

Aller au sommaire du numéro

Éditeur(s)

Globe, Revue internationale d'études québécoises

ISSN

1481-5869 (imprimé)

1923-8231 (numérique)

Découvrir la revue

Citer cet article

Tremblay, J. (2015). La contribution des conservateurs à la longue survie des organisations fascistes d'Adrien Arcand. Un élément d'explication. Globe, 18(1), 47-64. https://doi.org/10.7202/1037877ar
Résumé de l'article

De 1929 jusqu'à sa mort en 1967, Adrien Arcand assume la direction de l'Ordre patriotique des goglus (1929-1934), du Parti national social-chrétien (1934-1938) et du Parti de l'Unité nationale du Canada (1938-1940, 1947-1958, 1965-1967). Pendant ce temps, il publie plusieurs journaux, dont Le Goglu (1929-1933), Le Miroir (1929-1933), Le Chameau (1930-1932), Le Patriote (1933-1938), Le Fasciste canadien (1935-1938), Le Combat national (1938-1939), L’Unité nationale (1953-1958) et Serviam (1965-2001). Pour financer ses médias et ses mouvements politiques constamment déficitaires, Arcand se comporte comme un véritable mercenaire en rendant deux sortes de services aux conservateurs qui consentent à lui verser des subsides. Il leur offre en tout premier lieu ses talents d'éditorialiste en soutenant constamment leur politique dans ses journaux. Il demande, en second lieu, à ses disciples fascistes de militer en faveur de leur parti politique lors des campagnes électorales. C'est en concluant ce genre d'accords avec les principaux ténors du Parti conservateur du Canada et de l'Union nationale qu'Adrien Arcand a pu mener en toute impunité sa campagne antisémite et fasciste pendant plus de trente ans. 


\title{
LA CONTRIBUTION \\ DES CONSERVATEURS \\ À LA LONGUE SURVIE \\ DES ORGANISATIONS \\ FAS CISTES \\ D'ADRIEN ARCAND. \\ UN ÉLÉMENT \\ D'EXPLICATION
}

\section{JONATHAN TREMBLAY}

Université du Québec à Chicoutimi

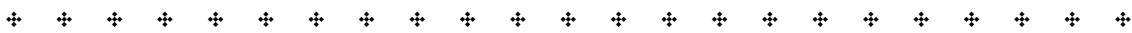

Résumé - De 1929 jusqu'à sa mort en 1967, Adrien Arcand assume la direction de l'Ordre patriotique des goglus (1929-1934), du Parti national social-chrétien (1934-1938) et du Parti de l'Unité nationale du Canada (1938-1940, 1947-1958, 1965-1967). Pendant ce temps, il publie plusieurs journaux, dont Le Goglu (1929-1933), Le Miroir (1929-1933), Le Chameau (1930-1932), Le Patriote (1933-1938), Le Fasciste canadien (1935-1938), Le Combat national (1938-1939), L'Unité nationale (1953-1958) et Serviam (1965-2001). Pour financer ses médias et ses mouvements politiques constamment déficitaires, Arcand se comporte comme un véritable mercenaire en rendant deux sortes de services aux conservateurs qui consentent à lui verser des subsides. Il leur offre en tout premier lieu ses talents d'éditorialiste en soutenant constamment leur politique dans ses journaux. Il demande, en second lieu, à ses disciples fascistes de militer en faveur de leur parti politique lors des campagnes électorales. C'est en concluant ce genre d'accords avec les principaux ténors du Parti conservateur du Canada et de l'Union nationale qu'Adrien Arcand a pu mener en toute impunité sa campagne antisémite et fasciste pendant plus de trente ans.
\end{abstract}




\section{$\div \quad \div$ \\ The Contribution of the Conservatives in the Long Survival of Adrien Arcand's Fascist Organizations : an Explanatory Element \\ One aspect of the Conservative Party's contributions \\ to the persistence of Adrien Arcand's fascist organizations}

Abstract - Between 1929 and his death in 1967, Adrien Arcand assumed the leadership of three organizations: the Ordre patriotique des goglus (1929-1934), the Christian National Socialist Party (1934-1938), and the National Unity Party (1938-1940, 1947-1958, 1965-2003). Over the same period, he published several newspapers, including Le Goglu (1929-1933), Le Miroir (1929-1933), Le Chameau (1930-1932), Le Patriote (1933-1938), Le Fasciste Canadien (1935-1938), Le Combat National (1938-1939), L’Unité Nationale (1953-1958), and Serviam (1965-2001). These publications and political movements consistently lost money. In order to finance them, Arcand behaved like a mercenary, providing two types of services to the Conservative Party: his skills as a columnist, which he consistently used to support Conservative policies in his newspapers, and the assistance of his fascist followers during election campaigns. In turn, the Conservatives agreed to subsidize Arcand's ventures. Negotiating this type agreement with key leaders of the Conservative Party of the Canada and the Union Nationale in Quebec gave Arcand the freedom to continue pursuing his anti-Semitic and fascist campaigns for more than thirty years.

$4+4 \div 4 \div 4 \div 4 \div 4 \div 4 \div 4 \div 4 \div 4 \div 4 \div$

De 1929 jusqu'à sa mort en 1967, Adrien Arcand ${ }^{1}$ parvient à mener une intense campagne antisémite et fasciste en dirigeant l'Ordre patriotique des goglus (1929-1934), le Parti national social-chrétien (19341938) et le Parti de l'Unité nationale du Canada (1938-1940, 1947-1958, $1965-1967^{2}$ ). Pendant ce temps, il détient ou dirige plusieurs journaux qui sont constamment déficitaires, dont Le Goglu (1929-1933), Le Miroir (19291933), Le Chameau (1930-1932), Le Patriote (1933-1938), Le Fasciste

$$
4+4
$$

1. Né à Montréal en 1899, Adrien Arcand devient journaliste en publiant tour à tour des articles dans les pages de La Patrie (1919), du Montreal Daily Star (1920) et de La Presse (1920-1929). En 1929, il participe à la fondation de l'Ordre patriotique des goglus, un groupe de pression nationaliste canadienfrançais. Cependant, au moment où le gouvernement de Louis-Alexandre Taschereau adopte la loi sur les écoles juives en 1930, Adrien Arcand deviendra pour le reste de sa vie un indéfectible antisémite. Puis, à partir de 1932, Adrien Arcand demande à rencontrer Kurt Ludecke, le principal émissaire du Parti national-socialiste des travailleurs allemands (NSDAP) en Amérique du Nord. C'est ce dernier qui le convainc de fonder un mouvement fasciste dans le but de mener sa campagne antisémite sur la scène politique. Ainsi, jusqu'à sa mort en 1967, Arcand demeurera un impénitent nazi (Jonathan TrEMBLAY, "Un accommodement déraisonnable? Une explication de la survie des organisations fascistes d'Adrien Arcand pendant l'entre-deux-guerres", mémoire de maîtrise (études et interventions régionales), Université du Québec à Chicoutimi, 2014, p. 1-27).

2. De 1967 à 2003, le successeur d'Arcand est Gérard Lanctôt. Le Parti de l'Unité nationale du Canada semble être disparu de la scène politique depuis 2003. 
canadien (1935-1938), Le Combat national (1938-1939), L'Unité nationale (1953-19583) et Serviam (1965-2001). Pourtant, Adrien Arcand ne dispose pas des moyens nécessaires pour financer ces organisations politiques et ces médias. En effet, de mai 1937 à février 1940, au moment où il est au sommet de sa carrière journalistique en étant rédacteur en chef de L'Illustration nouvelle, Arcand gagne un peu moins de cent dollars par semaine $e^{4}$.

Le chef fasciste québécois ne peut pas non plus compter sur les cotisations de ses militants pour éponger les déficits de ses organisations politiques, puisqu'ils ne sont pas assez nombreux à en faire partie. En fait, comme le confirment deux sources crédibles, tout au plus quelques milliers de personnes adhèrent à l'Ordre patriotique des goglus, au Parti national social-chrétien (PNSC) et au Parti de l'Unité nationale du Canada (PUNC).

Tout d'abord, les dirigeants du Congrès juif canadien (CJC), qui demandent à certains de leurs sympathisants d'infiltrer les organisations dirigées par Arcand, confirment que 5000 personnes militent au sein du PNSC lorsque ce parti est à son apogée en $1938^{5}$. L'autre source est celle des agents de la Gendarmerie royale du Canada (GRC), dont la mission consiste à observer de près les activités d'Adrien Arcand. D'après leurs estimations, le PUNC dispose en janvier 1940 de 7083 membres ${ }^{6}$.

Par conséquent, le chef fasciste québécois ne compte pas assez de cotisants pour financer ses entreprises politiques et journalistiques. Dans ce cas, comment Adrien Arcand a-t-il réussi à mener sa campagne antisémite et fasciste de 1929 à 1967 ? Nous soumettons l'hypothèse qu'Arcand n'a pu maintenir la viabilité de ses mouvements qu'en monnayant son appui à un politicien disposé à regarnir sa trésorerie constamment à sec. Ainsi, le leader antisémite s'engage à publier des éditoriaux résolument favorables au chef politique qui l'appuie, en plus d'ordonner à ses membres de le soutenir lors des campagnes électorales. Par contre, si ce politicien ne lui fournit plus de subsides, Arcand cesse de le soutenir dans ses journaux en arguant que celuici ne sert plus désormais les intérêts de la nation canadienne-française. Il

$$
+\div \div
$$

3. Jean-Louis LAPORTE, "Adrien Arcand déclare: guerre mondiale... ", Nouvelles illustrées, 2 mai 1965, p. 627 et PARTi de l'Unité nationale du CANada, Adrien Arcand devant le tribunal de l'histoire. Scandale à la Société Radio-Canada, Montréal, Parti de l'Unité nationale du Canada, 1983, p. 15.

4. BIBLIOTHĖQUE ET ARCHIVES NATIONALES DU QUÉBEC, FONDS DE LA FAMILLE ClÉMENT-ARTHUR DANSEREAU, Lettre d'Adrien Arcand à Eugène Berthiaume, 13 octobre 1939, p. 3.

5. David Rome, Clouds in the Thirties. On Anti-Semitism in Canada 1929-1939, a Chapter on Canadian Jewish History, vol. 5, Montréal, Canadian Jewish Congress, 1978, p. 18.

6. GENDARMERIE ROYALE DU CANADA, La loi et l'ordre dans la démocratie canadienne, Ottawa, Edmond Cloutier, 1952, p. 158. 
décide dans ce cas de se trouver une nouvelle source de financement en cherchant l'appui d'un autre politicien désireux de s'allier avec lui ${ }^{7}$. Il appert que cette stratégie fonctionne au sein des tories. Nous montrerons dans quelles circonstances Adrien Arcand parvient à obtenir le soutien des conservateurs provinciaux (1929-1930, 1932-1935) et fédéraux (1930-1932 et 1935), ainsi que l'appui de l'Union nationale (1935-1967).

\section{ADRIEN ARCAND DEVIENT HOUDISTE}

Le conservateur Camillien Houde, qui cumule les fonctions de chef de l'opposition provinciale et de maire de Montréal, a besoin de l'appui constant d'un journaliste montréalais pour assurer sa réélection à la mairie en 1930. Il est en effet parfaitement conscient que seulement sept des soixante-quatre journaux publiés au Québec soutiennent la politique des conservateurs ${ }^{8}$.

De son côté, dès le lancement des premiers numéros du Goglu et du Miroir en 1929, Adrien Arcand constate qu'il ne dispose pas des liquidités nécessaires afin d'assurer la pérennité de ses journaux. Il promet alors à Camillien Houde de toujours l'appuyer dans ses éditoriaux s'il consent à lui verser des subsides secrets ${ }^{9}$. Cette entente a rapidement été conclue, car le journaliste Arcand soutient constamment la politique de Houde à partir de la parution du troisième numéro du Goglu ${ }^{10}$.

Adrien Arcand tient compte de ce qui se passe à l'Hôtel de Ville pour dresser un portrait élogieux du maire conservateur. Il constate tout d'abord que la majorité des échevins siégeant au conseil municipal sont d'allégeance libérale. Il prétend ainsi que Houde ne peut pas mettre en place les réformes promises lors de la campagne électorale de 1928, car les conseillers libéraux s'opposent à tous les projets de loi qu'il propose. Arcand suggère donc à ses lecteurs de voter en faveur des candidats houdistes lors des élections municipales de 1930, car cela permettrait à Houde d'obtenir la majorité nécessaire à la réalisation de sa plate-forme électorale ${ }^{11}$.

$$
4+4
$$

7. Jonathan TREMBLAY, «Un accommodement déraisonnable? Une explication de la survie des organisations fascistes d'Adrien Arcand pendant l'entre-deux-guerres "..., op. cit., p. 5-6.

8. Marc La Terreur, Les tribulations des conservateurs au Québec. De Bennett à Diefenbaker, Québec, Les Presses de l'Université Laval, 1973, p. 71.

9. [s. a.], "Camillien Boude», L'Avenir du Nord, vol. 33, n 5, 20 décembre 1929, p. 12; [s. a.], « Houde demande 2500000 \$", Le Fouet, 31 octobre 1930, p. 7 ; Émile GOGLU, "Les six questions de Monsieur A. G. ", Le Goglu, vol. 1, no 13, 31 octobre 1929, p. 4 ; PARTI DE L’Unité NATIONALE DU CANADA, Adrien Arcand devant le tribunal de l'histoire..., op. cit., p. 8 et Robert RUMILLY, Histoire de la province de Québec, Montréal, Fides, 1959, p. 55.

10. Jean-François NADEAU, Adrien Arcand. Führer canadien, Montréal, Lux, 2010, p. 56.

11. Émile GOGLU, «Le dénommé Laprade", Le Goglu, vol. 1, n 33, 21 mars 1930, p. 2. 
Cependant, immédiatement après ces élections municipales montréalaises de 1930, les liens de collaboration entre le maire et le chef des goglus sont définitivement rompus, car ils se querellent pendant la campagne électorale à propos de l'affaire des écoles juives. Pour comprendre comment ces deux hommes se sont brouillés autour de cette question, il faut en premier lieu expliquer pourquoi certains Juifs désirent obtenir un réseau scolaire indépendant à Montréal.

\section{L'AFFAIRE DES ÉCOLES JUIVES}

Tout d'abord, en vertu de l'article 93 de la Constitution canadienne, seuls les catholiques et les protestants peuvent administrer les écoles publiques. Depuis 1903, le gouvernement du Québec considère que les Juifs sont des protestants «aux fins de l'éducation ${ }^{12}$ ".

Toutefois, au fil du temps, plusieurs parents juifs expriment leur désaccord face aux commissaires protestants. Tout d'abord, arguent-ils, ils paient leurs taxes scolaires alors qu'ils n'ont même pas le droit de siéger aux conseils d'administration des commissions scolaires dirigés par les tenants de l'Église réformée. Ensuite, disent-ils, l'enseignement est uniquement prodigué en fonction des besoins religieux et culturels des élèves protestants, et il n'est pas adapté aux réalités de leurs enfants.

Les commissaires rétorquent qu'il est normal que le cursus scolaire des écoles protestantes soit d'abord conçu pour les élèves protestants. Ils répliquent également que la valeur des propriétés juives est bien plus faible que celle des propriétés protestantes. Il est donc logique que ce soit uniquement des protestants qui siègent dans les conseils d'administration, puisque ce sont eux qui paient le plus de taxes scolaires.

Tout au long des années 1920, les Juifs poursuivent les commissaires protestants devant les tribunaux, mais cela ne règle en rien les litiges qu'ils ont avec eux. Plusieurs Juifs canadiens demandent alors au gouvernement provincial de créer un réseau scolaire juif à l'intérieur du système québécois. Cela leur permettrait ainsi de vivre leur foi et leur culture dans leurs propres écoles. Le gouvernement de Louis-Alexandre Taschereau réagit en adoptant le 4 avril 1930 un projet de loi où il est stipulé que les leaders de la communauté juive et les commissaires protestants ont un an pour trouver un terrain d'entente en vue d'intégrer les élèves juifs dans les écoles

$$
4+4
$$

12. QUÉBEC, STATUTS DU QUÉBEC, Loi amendant les lois concernant l'instruction publique relativement aux personnes professant la religion judaïque, Québec, Éditeur officiel, 1903, art. 1-7. 
réformées. Par contre, s'ils ne parviennent pas à conclure un accord au bout de ces douze mois, le gouvernement reconnaittra officiellement l'existence de la Commission des écoles juives de Montréal ${ }^{13}$.

Dès que ce projet de loi est sanctionné, Adrien Arcand analyse une foule de documents qui traitent des Juifs. Il souhaite ainsi publier dans ses journaux des arguments pertinents en vue de s'opposer à la loi sur les écoles juives. Finalement, après avoir mené ses recherches, Arcand justifie son opposition à cette législation en s'appuyant sur les publications de l'idéologue antisémite britannique Henry Hamilton Beamish. D'après ce dernier, les Juifs ourdissent depuis la résurrection de Jésus des complots dans le but de détruire la chrétienté, car ils ont conclu un pacte avec Satan en vue de dominer le monde. Il serait donc du devoir des chrétiens, soutient toujours Beamish, de se coaliser afin de lutter contre cette prétendue conjuration des Juifs à l'échelle internationale ${ }^{14}$. C'est sur la base de cette renversante théorie de Beamish qu'Adrien Arcand mènera, jusqu'à la fin de ses jours, une virulente campagne antisémite. En effet, comme il l'écrira de nombreuses années plus tard, Beamish a été «un de mes professeurs sur LA question [celle des Juifs] (il est le plus important après $\mathrm{M}^{\mathrm{gr}}$ Benigni et $\mathrm{M}^{\mathrm{gr}}$ Jouin) ${ }^{15}$ ».

De son côté, Camillien Houde comprend qu'Arcand est devenu infréquentable depuis que ce dernier a commencé à publier des articles antisémites. Cela ne veut pas dire que Houde soit favorable à la loi sur les écoles juives; au contraire, il se demande si le gouvernement ne veut pas accorder un traitement de faveur aux Juifs en leur permettant de se constituer un réseau scolaire indépendant de celui des catholiques et des protestants ${ }^{16}$.

$$
\div+\div
$$

13. Pour en savoir plus sur l'affaire des écoles juives : Arlette CORCOS, Montréal. Les Juifs et l'école, Sillery, Septentrion, 1997; David Rome, Canadian Jewish Archives on the Jewish School Question in Montreal 1903-1931, Montréal, Canadian Jewish Archives, 1975 ; Marc HÉBERT, «La presse de Québec et les Juifs 1925-1939. Le cas du Soleil et du Quebec Chronicle Telegraph", mémoire de maîtrise (histoire), Université Laval, 1994 et Robert Rumilly, "L'affaire des écoles juives (1928-1931) ", Revue d'histoire de l'Amérique française, vol. 10, $\mathrm{n}^{\circ} 2$, septembre 1956, p. 222-244.

14. Gisela C. Lebzelter, "Henry Hamilton Beamish and the Britons. Champions of Anti-Semitism ", Kenneth LunN, Richard C. Thurlow (dir.), British Fascism, Londres, Croom Helm Limited, 1980, p. 43-44 et 54 .

15. "Lettre d'Adrien Arcand au chanoine Georges Panneton, 4 novembre 1965 ", David PHILLIPS, Adrien Arcand ou... la vérité retrouvée, Saint-Léonard, Béluga, 2002, p. 329. Par ailleurs, dans une entrevue qu'il accorde en 1937 au journaliste David Martin, Arcand estime que Beamish est l'un des pionniers du mouvement antisémite (David MARTIN, "Interview with Adrien Arcand», Time Magazine, $\mathrm{n}^{\circ}$ 138, octobre-novembre 1937, p 9-10).

16. [s. a.], "L'Opposition provinciale demandera le rappel de la loi des écoles juives", Le Devoir, 8 octobre 1930 et [s. a.], «M. Houde demandera le rappel de la loi des écoles juives », L'Action catholique, 9 octobre 1930 . 
Houde réalise cependant qu'il ne pourra pas se faire réélire s'il se brouille avec une frange aussi importante de son électorat ${ }^{17}$. Les Juifs forment à ce moment-là la troisième communauté en importance de Montréal, derrière celle des Canadiens français et des Britanniques. Par conséquent, en 1930, il décide de condamner publiquement la vague antisémite soulevée par Arcand :

Lorsque j’ai été élu maire de Montréal, il y a deux ans, j’ai déclaré que j'étais le représentant de tous, et que je ne désirais pas voir soulever des questions de race sous mon régime. Il en surgit une présentement à Montréal, je la dénonce sur l'heure. S'il me faut soulever les races les unes contre les autres pour jouir du pouvoir, j'y renonce. Je ne veux pas plus du rôle de M. Anderson, de la Saskatchewan [qui venait pratiquement d'éliminer l'enseignement public de la langue française et de la religion catholique dans sa province] que celui de M. Norris, du Manitoba [qui a fait la même chose]. L'intérêt des miens à travers tout le pays exige de moi cette attitude ${ }^{18}$.

Puis, quelque temps après sa réélection, Houde permet aux Juifs d'ouvrir leurs magasins le dimanche s'ils ferment boutique en pratiquant le sabbat le samedi ${ }^{19}$. Pour Adrien Arcand, cela est la goutte d'eau qui fait déborder le vase. En effet, tout le long des années 1930, il se vengera de l'affront que lui a fait subir Camillien Houde en appuyant dans ses journaux un grand nombre de ses adversaires politiques ${ }^{20}$.

Parallèlement, toujours dans l'optique d'assurer la survie de ses entreprises politiques et journalistiques, Adrien Arcand réussit à obtenir du financement des conservateurs fédéraux. C'est ainsi qu'il deviendra l'un des plus vibrants chantres du premier ministre canadien Richard Bedford Bennett de 1930 jusqu'en 1935.

$$
\div+\div
$$

17. Hughes THÉORÊT, Le docteur Lalanne. Le faiseur d'anges à la croix gammée, Québec, Éditions GID, 2011, p. 73.

18. Robert LÉVESQUE et Robert MIGNER, Camillien et les années vingt suivi de Camillien au goulag, Montréal, Éditions des Brûlés, 1978, p. 109.

19. David ROME, Clouds in the Thirties..., op. cit., p. 88-89.

20. Ibid., p. 34-35; Hertel LA RoQue, Camillien Houde. Le p'tit gars de Sainte-Marie, Montréal, Éditions de l'Homme, 1961, p. 74 et p. 81-82; Louis-Martin TARD, Camillien Houde. Le Cyrano de Montréal, Montréal, XYZ, 1999, p. 113; Raymond OUIMET, L'affaire Tissot. Campagne antisémite en Outaouais, Montpellier, Écrits des Hautes-Terres, 2006, p. 109 et Robert RUMILly, Histoire de la province de Québec..., op. cit., tome 33, p. 51 et tome 34, p. 24-25. 


\section{ARCAND CONCLUT DEUX ENTENTES AVEC BENNETT}

Depuis que le gouvernement de Robert Laird Borden leur a imposé la conscription en 1917, les Canadiens français sont peu enclins à voter pour le Parti conservateur du Canada. R. B. Bennett, qui veut remporter les élections législatives de 1930, souhaite mener durant l'été une intense campagne électorale afin de convaincre les Québécois de voter en faveur des tories. Il demande donc à Adrien Arcand d'organiser des réunions publiques et de publier des messages de propagande en faveur des conservateurs. En retour, Bennett promet de lui verser $25000 \$$ et de rembourser tous les frais qu'il contracterait au cours de la campagne électorale, s'il parvenait à faire élire douze députés tories au Québec.

Le chef antisémite québécois consent à cette offre, car ses organisations sont toujours déficitaires. Il publie donc 100000 tracts, en plus d'organiser 104 réunions en faveur du parti et d'imprimer 80000 exemplaires du Goglu et du Miroir ${ }^{21}$. Ces efforts portent leurs fruits, puisqu'à l'issue du scrutin du 28 juillet 1930, les conservateurs remportent les élections en obtenant à travers tout le Canada 137 sièges, dont 24 au Québec.

Adrien Arcand a donc réussi à remplir ses engagements envers Bennett $^{22}$. Par conséquent, ce dernier doit rembourser les frais encourus par les goglus pour financer la campagne électorale des conservateurs au Québec. Ces frais s'élèvent à 47900 \$. Le nouveau premier ministre canadien doit en outre lui allouer les $25000 \$$ garantis initialement avant la campagne électorale. Arcand expédie donc à Bennett une facture dont la somme totale s'élève à $72900 \$$. Richard Bedford Bennett décide cependant de ne lui verser que $18000 \$ 23$.

$$
4 \div
$$

21. ARChives nationales du CANAdA, AdRIEN ARCAND - INTERVIEW REQUeSTS, 1930-1932, Mémorandum d'Adrien Arcand et de Joseph Ménard à Richard Bedford Bennett, 14 janvier 1931.

22. Ce n'est pas seulement l'intense activité publicitaire menée par Arcand en faveur des conservateurs qui a permis à ces derniers de remporter les élections. En 1930, les effets du krach boursier de 1929 commencent à se faire sentir au Canada et les électeurs manifestent leur mécontentement à l'égard du premier ministre sortant William Lyon Mackenzie King en votant pour Bennett. À ce sujet, voir : Claude LARIVIÈRE, "Crise économique et contrôle social. Le cas de Montréal (1929-1937) ", mémoire de maîtrise (service social), Université de Montréal, 1976; H. Blair NEATBY, The Politics of Chaos. Canada in the Thirties, Toronto, Macmillan of Canada, 1972 et Pierre BERTON, The Great Depression. 1929-1939, Toronto, McClelland and Stewart, 1990.

23. Julian SHER, White Hoods. Canada's Ku Kux Klan, Vancouver, New Star Books, 1983, p. 63 ; Michèle LAROSE, «Les jeunesses patriotes et La Nation. Un courant politique d'extrême-droite au Québec, 19341939 ", mémoire de maîtrise (histoire), Université du Québec à Montréal, 1984, p. 47-48; Michelle MCBRIDE, "From Indifference to Internment. An Examination of RCMP Responses to Nazism and Fascism in Canada from 1934 to 1941 ", mémoire de maîtrise (histoire), Memorial University, 1997, p. 62-64 et Stéphane MORISSET, "Adrien Arcand. Sa vision, son modèle et la perception inspirée par son programme », mémoire de maîtrise (histoire), Université Laval, 1995, p. 36-38. 
Le chef du Parti conservateur du Canada n'a donc pas respecté l'accord conclu avec le leader antisémite. Il lui promet cependant de lui allouer au cours des prochaines années une série de petits versements afin de lui payer le reste de son dû. Bennett agit ainsi, car il veut s'assurer qu'Adrien Arcand sera toujours fidèle à son parti. Ainsi, si Arcand se mettait un jour à ne plus appuyer la politique des conservateurs dans ses éditoriaux, R. B. Bennett pourrait réagir rapidement en lui coupant les vivres

Cette prudence que le premier ministre manifeste à son égard est de bon aloi, puisqu'Arcand connaît quelques mois plus tard de sérieux ennuis juridico-financiers. Tout d'abord, à la demande des leaders de la communauté juive, presque tous les commerçants consentent à retirer leurs annonces des pages du Goglu et du Miroir. Ils craignent en effet que leurs clients ne relient leurs produits à ces publications haineuses. Des dizaines de personnes poursuivent également Arcand devant les tribunaux pour cause de diffamation. Puis, durant les élections législatives provinciales de 1931, des opposants tentent de museler Adrien Arcand en incendiant l'imprimerie des journaux dont il est le rédacteur en chef. Cependant, comme ce dernier réussit à reconstruire ses installations, les adversaires des goglus répliquent en y allumant le feu à deux autres reprises. Le dernier incendie criminel, qui a eu lieu en 1933, a complètement détruit l'atelier ${ }^{24}$.

Arcand est au bord de la faillite. Il écrit tout d'abord une kyrielle de lettres au premier ministre Bennett afin de lui signaler que ses entreprises politiques et journalistiques cumulent une dette de plus de $50000 \$$. Il exige ensuite qu'il respecte la promesse qu'il lui avait faite durant la campagne électorale de 1930 en lui remboursant le reste de son dû. Il lui demande enfin à de nombreuses reprises de lui accorder une audience pour régler cette affaire, mais Bennett refuse catégoriquement de le recevoir à son bureau à partir d'octobre $1931^{25}$.

Le chef du Parti conservateur du Canada a de bonnes raisons d'agir ainsi envers son organisateur électoral, puisque ce dernier lui a montré qu'il est devenu infréquentable avec son discours d'extrême-droite. En effet, Arcand a cherché à convertir son mécène à son idéologie politique en lui

$$
\div+\div
$$

24. David PHILlips, Adrien Arcand ou..., op. cit., p. 17 ; David Rome, Clouds in the Thirties..., op. cit., vol. 1, p. 114 et Martin RoBIN, Le spectre de la droite, Montréal, Balzac-Le Griot éditeur, 1998, p. 123124.

25. ARChives nationales du CAnada, Bureau du Premier ministre du Canada, Requests-Interview Requests-Adrien Arcand, 1930-1932, bobine de microfilm M-1326, MG26-K et ARCHIVES NATIONALES DU CANADA, Documents de Bennett (Conservative Party-Quebec-Montreal), bobine de microfilm M1248, MG26-K. 
acheminant de nombreux documents antisémites et fascistes. Il lui a tout d'abord expédié un exemplaire du Fascist d'Arnold Spencer Leese, avant de lui adresser plusieurs articles antisémites italiens, autrichiens, bohémiens, hongrois et français. Le leader de l'Ordre patriotique des goglus a aussi expédié au bureau du premier ministre un article ayant été publié dans le Daily Mail par le vicomte Rothermere ${ }^{26}$. C'est également Arcand qui a invité le vicomte Léon de Poncins à adresser un questionnaire à Bennett dans le but de tester ses convictions antisémites ${ }^{27}$.

Après avoir reçu tous ces colis, R. B. Bennett en conclut qu'il vaut mieux se débarrasser d'un allié aussi encombrant. Il décide alors de ne plus lui verser de subsides et d'attendre que ses entreprises politiques et journalistiques fassent faillite. Celle-ci survient au printemps 1933, lorsqu'Adrien Arcand publie les derniers numéros du Goglu et du Miroir ${ }^{28}$.

Plus tard, un peu avant les élections législatives de 1935, R. B. Bennett constate qu'il est devenu impopulaire à travers l'ensemble du Canada. Il faut dire que son gouvernement a été incapable de résoudre les nombreux problèmes socioéconomiques engendrés par la crise de 1929. Il est donc prêt à se tourner vers n'importe qui pour organiser sa campagne électorale, car les personnes désireuses de militer en faveur de son parti se font rares.

De son côté, Adrien Arcand vient tout juste de fonder le PNSC un an plus tôt. Il comptait déposer sa candidature lors de cette campagne électorale, mais il estime maintenant qu'il manque de liquidités afin de la mener à bien. Profitant de ce contexte favorable, Bennett conclut un nouvel accord avec Adrien Arcand. Il promet de lui verser 27000 \$ s'il parvient à faire élire un nombre raisonnable de candidats tories dans la province ${ }^{29}$.

Durant la campagne électorale, Arcand publie des articles dithyrambiques à l'égard du premier ministre sortant. Il le présente comme un fervent chrétien, un ami de la loi et de l'ordre, un virulent anticommuniste et un homme qui a bien représenté les intérêts du Canada en participant avec

$$
\div+\div
$$

26. ARCHIVES PUBLIQUES DU CANADA, Bureau du premier ministre du Canada, Requests-Interview Requests-Adrien Arcand, 1930-1932, bobine de microfilm M-1326, MG26-K et Mémo d'Adrien Arcand à Arthur W. Merriam, octobre 1930.

27. ARChIVES NATIONALES DU CANADA, Documents de Bennett, bobine de microfilm M1474, MG26K, 1935-1938, p. 519238-519359.

28. Martin RoBIN, Le spectre de la droite..., op. cit., p. 125 et 134.

29. ARChives nationales du CANAdA, Documents de Bennett (Conservative Party, Organization Quebec), bobine de microfilm M-1115, MG26K, Lettre de M. T. Bérubé à René Campion, 27 septembre 1935 et PARTI DE L'Unité nATIONALE DU CANADA, Adrien Arcand devant le tribunal de l'histoire..., op. cit., p. 9. 
brio aux conférences impériales de 1930 et de 1932. Cette rengaine, qu'il répète constamment depuis les débuts de son association avec les conservateurs fédéraux en 1930, ne produit cette fois aucun effet sur l'ensemble des électeurs. Le jour du scrutin, seulement cinq candidats tories sont élus au Québec. Cette fois, les conservateurs rompent définitivement leur alliance avec Arcand, car ils constatent que ce dernier ne peut pas convaincre les Canadiens français de voter en leur faveur ${ }^{30}$.

Cependant, ce ne sont pas tous les tories qui ont rompu leurs liens d'amitié avec Arcand. Au Québec, les troupes de l'Union nationale vont prendre le relais de celles de Bennett pour lui apporter de l'aide, car le chef fasciste consent, de 1932 jusqu'à sa mort en 1967, à militer en faveur des unionistes. Adrien Arcand doit d'ailleurs deux choses à ce parti politique: l'immunité, en ne se faisant pas arrêter pour cause de sédition avant la Deuxième Guerre mondiale, et de l'aide financière, surtout après 1945.

\section{L'UNION NATIONALE A EMPÊCHÉ ARCAND DE CREVER Maurice Duplessis protège le chef fasciste}

En 1932, Camillien Houde choisit de quitter ses fonctions à la tête du Parti conservateur du Québec. Il demande ensuite à l'ensemble des militants tories de voter en faveur d'Onésime Gagnon lors de la course à la chefferie du parti à l'automne 1933. Pour sa part, Maurice Duplessis choisit de défier Houde en déposant sa candidature en vue de lui succéder.

Arcand profite de ce contexte pour se venger de Houde. Il appuie inconditionnellement Duplessis en ordonnant à ses goglus d'organiser sa campagne pendant la course à la chefferie. Puis, une fois que le député de Trois-Rivières est élu chef de cette formation politique à l'automne 1933, Adrien Arcand le soutient constamment en le décrivant de manière élogieuse dans ses éditoriaux. Ensuite, lorsque Maurice Duplessis fonde l'Union nationale en 1935, Arcand demande à ses partisans fascistes de l'aider en appuyant tous les candidats unionistes. En fait, Arcand soutiendra jusqu'au jour de sa mort l'Union nationale, qui le lui rendra très bien en retour ${ }^{31}$.

$$
4+4
$$

30. David MARTIN, «Interview with Adrien Arcand»..., op. cit., p. 8.

31. Alfred HARDY, Patronage et patroneux. Un témoignage inédit de l'ancien directeur général des achats du Québec, Montréal, Éditions de l'Homme, 1979, p. 136; [s. a.], "Arcand à Ottawa avec l'aide de Duplessis? ", Vrai, vol. 3, no 11, 10 novembre 1956, p. 1-2 ; [s. a.], "Arcand conspire à Lanoraie!", Vrai, vol. 3, n 4, 22 septembre 1956, p. 1 et 10 ; ARCHIVES DU CONGRÈS JUIF CANADIEN, Mémorandum de H. M. Caiserman à Hayes, 5 décembre 1946 et 19 décembre 1946; David ROME, Clouds in the Thirties..., op. cit., vol. 5, p. 40 ; Martin ROBIn, Le spectre de la droite..., op. cit., p. 136; Michèle LAROSE, «Les jeunesses patriotes et La Nation. Un courant politique d'extrême-droite au Québec, 1934-1939»..., op. cit., p. 48; Michelle MCBRIDE, «From Indifference to Internment. An Examination of RCMP 
Tout d'abord, à partir de la fondation de leur parti en 1935 jusqu'au décès d'Adrien Arcand en 1967, les unionistes seront toujours disposés à attribuer divers travaux d'édition et de traduction au leader d'extrêmedroite $^{32}$. Comme le dit de manière ironique Alfred Hardy, le directeur général des achats du Québec de 1937 à 1959: «Seuls les représentants des quotidiens opposés au régime n'obtenaient pas de chèques "pour traduction, recherche et interprétation de documents" 33 ".

De plus, en faisant adopter la loi du cadenas en 1937, le gouvernement de Duplessis encadre étroitement toutes les activités politiques des communistes, mais il ne fait rien pour empêcher Arcand de mener sa campagne antisémite et fasciste. Le seul fait de ne pas l'inquiéter constitue en soi un appui ${ }^{34}$.

Cette lune de miel avec le parti de Maurice Duplessis s'obscurcit cependant à partir de 1937, car plusieurs journalistes effraient leurs lecteurs en décrivant les activités menées par les légionnaires, ces disciples d'Arcand qui arborent la chemise bleue et qui s'entraînent hebdomadairement dans les locaux du PNSC 35 .

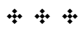

Responses to Nazism and Fascism in Canada from 1934 to 1941 ».., op. cit., p. 67 ; Réal CAUX, «Le Parti national social-chrétien. Adrien Arcand, ses idées, son œuvre et son influence", mémoire de maîtrise (sciences politiques), Université Laval, 1958, p. 70 et S, "Embryo Fascism in Quebec », Foreign Affairs, vol. $16, \mathrm{n}^{\circ} 3$, avril 1938, p. 457.

32. ARCHIVES DU CONGRÈS JUIF CANADIEN, Rapport préliminaire relativement à Adrien Arcand et à sa candidature dans le comté fédéral de Berthier-Maskinongé-Delanaudière, 9 août 1953, p. 4; Françoise CÔTÉ, "Fasciste d'un autre âge. Adrien Arcand", MacLean Magazine, vol. 1, mai 1961, p. 48 ; Kenneth G. WRIGHT, "Stronger than Ever Here", Is Arcand's Fascist Boast", The Montreal Gazette, 23 février 1947 ; David PHILlips, Adrien Arcand ou..., op. cit., p. 578 et [s. a.], "Les fascistes se terrent à St-Alphonse d'Youville", Vrai, 10 février 1957, p. 5.

33. Alfred HaRdy, Patronage et patroneux..., op. cit., p. 72.

34. David MARTIN, "Interview with Adrien Arcand»..., op. cit., p. 8; David ROME, Clouds in the Thirties..., op. cit., vol. 5, p. 40 ; Hughes THÉORÊT, «La campagne antisémite d'Adrien Arcand d'aprèsguerre. 1945 à 1967 ", mémoire de maîtrise (histoire), Université d'Ottawa, 2009, p. 71 et Stanley R. BARRETT, "Fascism in Canada", Contemporary Crisis, no 8, 1984, p. 352.

35. En ordre de parution, quelques-uns de ces articles: "Quebec Fascism», Canadian Forum, vol. 17, n ${ }^{\circ} 195$, avril 1937, p. 4-5; Télesphore-Damien BOUCHARD, "Qu'attend M. Duplessis pour agir? », En avant!', 26 novembre 1937 ; "Fascist Crowd Court ", Montreal Daily Star, décembre 1937; Ken W. MAC TAGGART, "Largent Fascist Unit is Led from Quebec », Globe and Mail, 2 décembre 1937; Jean-Charles HARVEY, "Qu'on arrête ces gangsters! ", Le Jour, 4 décembre 1937; David GRIFFIN, "Montreal Fascist Leader Aspires to Dictatorship in Canada ", Toronto Daily Star, 10 février 1938; S, "Embryo Fascism in Quebec»..., op. cit., p. 454-466; Frederick EDWARDS, "Fascism in Canada", Maclean's Magazine, vol. 51, n' 10, 1938, p. 66-69; Frederick EDWARDS, "Fascism in Canada. Part Two", Maclean's Magazine, $1^{\text {er }}$ mai 1938, p. 15 et 30 ; "Don't Be a Fascist Fool!", Canadian Magazine, vol. 89, n 2, mai 1938 ; "Does Canada Need Fascism?", The Country Guide and Nor-West Farmer, juillet 1938, p. 11 et 44 ; "Organisation de ces réunions fascistes à Kingston et à Toronto ", La Presse, no 97, $1^{\text {er }}$ juillet 1938 ; "Advertising Antidemocracy", The Montreal Standard, 2 juillet 1938; FlAMBEAU, "De profundis du fascisme à Kingston et à Toronto", L'Autorité, 9 juillet 1938; "Ban Fascist Uniforms", The Tattler Review, vol. 1, $\mathrm{n}^{\circ}$ 13, 16 juillet 1938; "Fascism in Quebec", The New Statesman and Nation, 27 août 1938, p. 304-305; John HOARE, "Swastika over Quebec. Arcand Meeting", Saturday Night, 9 septembre 1939 et "Arcand's Party Says Openly It Would End Democracy", The Standard, vol. 34, $\mathrm{n}^{\mathrm{o}} 22,1^{\mathrm{er}}$ juin 1940 , p. 1 . 
À la suite de la publication de ces reportages, les Canadiens s'inquiètent réellement pour la sécurité de leur pays, car ils craignent que les nazis demandent aux membres du PNSC de commettre des actes de sabotage en vue d'aider l'Allemagne à envahir le $\mathrm{Canada}^{36}$. Maurice Duplessis comprend à partir de ce moment-là qu'il ne pourra plus protéger Arcand comme il le faisait auparavant.

\section{Adrien Arcand est écroué pendant la Seconde Guerre mondiale}

Dans ce contexte, le premier ministre Duplessis juge qu'il vaut mieux prendre ses distances avec Adrien Arcand. Du moins publiquement. Il le fait en prononçant ce discours en 1938 :

Arcand n'a jamais été, n'est pas et ne sera jamais mon porte-parole, il n'est pas lié avec moi, ni avec mon parti. [...] M. Arcand est le chef du Parti fasciste, lequel non seulement ne m'appuie pas, mais est mon ennemi déclaré. [...] Je ne m'inquiète toutefois pas à son sujet, car le fascisme n'exerce aucun attrait sur les Canadiens français. M. Arcand est un fanatique, il a des visions et personne ne doit le prendre au sérieux. De plus, je puis ajouter que, pour ma part, je suis un bon catholique, mais je ne suis pas plus catholique que le pape et le pape a récemment déclaré ouvertement la guerre au fascisme et à l'antisémitisme, et c'est le but que je poursuis personnellement [je traduis $]^{37}$.

Pour sa part, durant l'été 1939, le gouvernement fédéral se prépare à déclarer la guerre à l'Allemagne. Le 31 août, le premier ministre canadien William Lyon Mackenzie King forme le Comité interministériel consultatif sur l'internement, destiné à enquêter sur les activités subversives au Canada. Trois jours plus tard, le Comité se prononce au sujet des mouvements fascistes dirigés par Adrien Arcand. Il estime qu'Arcand ne représente pas une menace à la sécurité nationale, car les adhérents du PUNC jurent allégeance au Canada et non pas à l'une ou l'autre des puissances ennemies.

Par ailleurs, au moment où le Canada déclare la guerre à l'Allemagne le 10 septembre 1939, la Loi sur les mesures de guerre et les Règlements concernant la défense du Canada entrent en vigueur. Le gouvernement dispose donc de tous les outils légaux pour procéder à la dissolution du PUNC,

$$
4+4
$$

36. Clifford W. Harvison, The Horsemen, Montréal/Toronto, McClelland and Stewart, 1967, p. 86; Gregory Sean Kealey et Reginald Whitaker, R.C.M.P. Security Bulletins. The Depression Years, Part V. 1938-1939, St. John's, Committee on Canadian Labour History, 1997, p. 2.

37. [s. a.], "Maurice Duplessis. Disavows Fascism. L'Illustration and Arcand!», The Canadian Jewish Chronicle, 28 octobre 1938, p. 11. Je n'ai pas consigné dans mes notes personnelles les citations originales, car je les ai traduites au fur et à mesure que je dépouillais les documents dans les centres d'archives. 
mais il ne le fait pas. Il demande plutôt au capitaine Innis, un officier de la GRC, de signaler à Adrien Arcand qu'il lui est désormais interdit de faire de la politique durant les hostilités ${ }^{38}$.

Arcand ne respecte pas cette consigne des autorités. Le 20 septembre 1939, il organise un rassemblement au Marché Saint-Jacques, où il prétend qu'il va prendre le pouvoir lors des prochaines élections. En outre, en octobre de la même année, il aide les candidats unionistes à mener leur campagne lors des élections législatives provinciales. Rien n'indique que le premier ministre Duplessis, qui agit également à titre de procureur général du Québec, ait demandé à son organisateur de respecter la consigne du capitaine Innis en ne faisant pas de politique en temps de guerre. Par ailleurs, à partir du 2 janvier 1940, Arcand s'enhardit en organisant des réunions clandestines dans les maisons de ses militants, en plus d'imprimer dans un sous-sol du boulevard Saint-Laurent le pamphlet antisémite The Jews and the War ${ }^{39}$.

Les policiers fédéraux réagissent alors en menant, du 23 au 27 mai 1940, une série de perquisitions dans les locaux du PUNC. Ils saisissent notamment une kyrielle de documents qui attestent qu'Adrien Arcand communique régulièrement avec de nombreux chefs fascistes à l'étranger. Ernest Lapointe, le ministre fédéral de la Justice, entame alors des procédures judiciaires en vue de procéder à la dissolution des mouvements fascistes dirigés par Adrien Arcand. Le 30 mai, la GRC arrête Arcand et quelques cadres de son parti. Le 4 juin, Lapointe annonce à la Chambre des communes qu'il interdit à quiconque d'adhérer au PUNC. Enfin, le 21 juin 1940, il décide de faire interner Arcand et dix de ses principaux lieutenants ${ }^{40}$ pour toute la durée du conflit ${ }^{41}$.

\section{Duplessis collabore de nouveau avec Arcand}

Une fois que la Seconde Guerre mondiale est terminée, en 1945, Adrien Arcand est libéré et obtient de nouveau tous ses droits. Il continue donc de mener sa campagne antisémite et fasciste en remettant sur pied le PUNC en 1947. De son côté, Maurice Duplessis consent toujours à lui rendre quelques

$$
+4 \div
$$

38. Michelle MCBRIDE, "From Indifference to Internment. An Examination of RCMP Responses to Nazism and Fascism in Canada from 1934 to 1941 »..., op. cit., p. 171-176.

39. Ibidem.

40. Ces dix lieutenants sont: Henri Arcand, Maurice Scott, Noël Décarie, G. R. Barck, Paul Giguère, Marius Gatien, Hughes Clément, Joseph C. Farr, John Lorimer et Léo Brunet.

41. OTTAWA, DOMINION DU CANADA, Compte-rendu officiel des débats de la Chambre des communes. Sixième session, dix-huitième législature et première législature et première session, dix-neuvième législature, 1940, Ottawa, Edmond Cloutier, 1940, p. 1012. 
services, car Arcand recommence à lui prêter main-forte pendant les campagnes électorales.

À titre d'exemple, Lucien Giguère, le beau-frère d'Adrien Arcand, fait en 1947 une collecte de fonds auprès des membres du PUNC afin de permettre à Arcand de racheter le Melody Club, un bar de la rue Sherbrooke à Montréal. Giguère veut en effet lui assurer un revenu stable pour lui permettre de poursuivre sa campagne antisémite ${ }^{42}$.

Le premier ministre Duplessis donne son aval à cette transaction en concluant un accord avec Adrien Arcand. C'est en effet le gouvernement du Québec qui alloue les permis d'alcool aux commerçants. Le député de Trois-Rivières permet ainsi au leader fasciste d'obtenir son permis, à condition qu'il ne gêne pas l'Union nationale avec ses discours d'extrême-droite. Les dirigeants du CJC certifient l'existence de cet accord :

A. [le nom de code des Juifs pour désigner la personne qui espionne les mouvements d'Arcand en leur nom] m'a informé que la première réunion de masse d'Arcand a fait une très mauvaise impression à Québec, et il m'a dit que si Arcand continue ainsi en faisant tant de publicité négative, le permis du Melody Club sera révoqué. C'est probablement la raison, a expliqué A., pour laquelle le premier ministre du Québec a averti Arcand de se modérer [je traduis] ${ }^{43}$.

\section{Durant les années 1960, Arcand continue à recevoir de l'aide de l'Union nationale}

Après la mort de Maurice Duplessis en 1959, de nombreux militants unionistes assistent à des conférences d'Adrien Arcand, en plus de s'abonner à ses journaux et d'acheter ses livres. C'est le cas du conseiller législatif Gérald Martineau ${ }^{44}$, du député provincial Yves Gabias ${ }^{45}$ et de Joseph-Ernest Laforce $^{46}$, un membre de la Commission du service civil de la province de Québec.

$$
4+4
$$

42. En ordre d'apparition: ARChIVES Du CONGRÈs JUIF CANADIEn, Mémo de H. M. Caiserman à Hayes, 19 décembre 1946 et ARCHIVES Du CONGrès JuIf CANADIEn, Lettre de H. M. Caiserman à Saul Hayes, 18 mai 1948.

43. ArChives du Congrès Juif Canadien, Mémorandum de Saul Hayes et de H. M. Caiserman, 19 novembre 1947.

44. ARChives nationales du CANADA, FondS AdRIEN ARCAND, Lettre de J. Ernest Laforce à Adrien Arcand, vol. 2, 3 mars 1960.

45. Archives nationales du Canada, Fonds AdRIEn ARCAND, Lettre d'Yves Gabias à M. et à $M^{\text {me }}$ Arcand, vol. 2, 6 avril 1961.

46. ARCHIVES NATIONALES DU CANADA, FONDS ADRIEN ARCAND, Lettre de J.-Ernest Laforce à Adrien Arcand, vol. 3, 7 août 1966 et ARCHIVES NATIONALES DU CANADA, FONDS ADRIEN ARCAND, Lettre de J.-Ernest Laforce à Adrien Arcand, vol. 3, 17 août 1966. 
En 1960, Adrien Arcand doit se faire opérer. Gérald Martineau, qui agit aussi à titre de trésorier de l'Union nationale, consent à lui verser cinq cents dollars, soit la moitié des coûts de son opération ${ }^{47}$. Martineau est si souvent généreux à l'égard d'Arcand que ce dernier lui écrit en 1963 : "cher M. Martineau (le seul, avec le Grand Disparu [Duplessis], à qui j'ai dû de ne pas crever littéralement ${ }^{48}$ ".

Enfin, tout au moins en 1962, des cadres de l'Union nationale consentent à soutenir le mouvement fasciste d'Adrien Arcand par l'entremise d'Eugène Doucet, un entrepreneur unioniste de Trois-Rivières ${ }^{49}$.

En somme, c'est uniquement grâce aux politiciens conservateurs qui l'ont rémunéré qu'Adrien Arcand a pu mener pendant plus de trois décennies une campagne antisémite et fasciste au Canada. Tout d'abord, dès le lancement des premiers numéros du Goglu et du Miroir en 1929, Arcand constate que ces hebdomadaires ne seront jamais rentables. Il offre donc à Camillien Houde de toujours le soutenir dans ses éditoriaux s'il consent à regarnir la trésorerie constamment asséchée de son organisation. Houde accepte cette proposition, car il sait que seulement sept des soixante-quatre journaux édités au Québec appuient la politique des conservateurs.

Cette association entre Houde et Arcand prend brusquement fin en 1930, à partir du moment où Arcand commence à mener sa campagne antisémite. Le maire de Montréal réagit aussitôt en cessant de verser des subsides à la trésorerie de l'Ordre patriotique des goglus et en condamnant publiquement les dérives racistes de son organisateur électoral. Adrien Arcand voudra par la suite se venger de cet affront que lui a fait Camillien Houde en soutenant plusieurs de ses adversaires politiques à la mairie.

Pendant ce temps, dans l'arène politique fédérale, Bennett recourt lui aussi aux services d'Adrien Arcand. Ainsi, Bennett consent à verser $25000 \$$ à Arcand si ce dernier parvient à faire élire douze députés conservateurs au Québec. Finalement, à l'issue du scrutin du 28 juillet 1930, les conservateurs remportent les élections en obtenant à travers tout le pays 137 sièges, dont 24 au Québec. Le chef tory ne respecte pas l'accord qu'il a conclu avec Adrien Arcand, car il prend graduellement conscience que son organisateur électoral devient un allié politique gênant avec ses discours

$$
+4+4
$$

47. ArChives nationales du Canada, Fonds Adrien ARCAND, Lettre de J. Ernest Laforce à Adrien Arcand, vol. 2, 3 mars 1960.

48. Archives nationales du Canada, Fonds Adrien ARCAND, Lettre d'Adrien Arcand à Gérald Martineau, vol. 2, 3 juillet 1963.

49. [s. a.], "Lettre de Pierre Gravel à Adrien Arcand», 11 octobre 1962, David PHILLIPS, Adrien Arcand ou..., op. cit., p. 190. 
d'extrême-droite. Il cherche donc à se débarrasser de lui et il en a l'occasion lorsqu'Arcand lui signale qu'il a contracté une dette de plus de 50000 \$. Bennett refuse alors de lui rembourser ses dettes, car il sait que cela l'acculera à la faillite, ce qui arrive au printemps 1933 .

Toutefois, contrairement à Camillien Houde, Bennett n'a pas définitivement abandonné Arcand après avoir découvert que ce dernier était un antisémite. En effet, durant les élections législatives de 1935, Bennett conclut avec Arcand une entente semblable à celle de 1930. C'est uniquement après la défaite du Parti conservateur en 1935 que ces deux hommes se résoudront à rompre complètement leurs liens.

Par ailleurs, l'attitude des unionistes québécois est plus constante que celle des conservateurs fédéraux. En effet, à partir de 1932 jusqu'à sa mort en 1967, Adrien Arcand soutient ouvertement Maurice Duplessis et tous ses successeurs à la tête de l'Union nationale. Cela signifie que, même après la Seconde Guerre mondiale, les cadres du parti fondé par Duplessis continuent à financer le principal chef nazi du Canada en lui allouant un permis d'alcool, en achetant ses publications et en lui octroyant plusieurs contrats en vue de mener divers travaux d'édition et de traduction pour le gouvernement.

À cet égard, il est très clair que Maurice Duplessis n'a éprouvé aucun scrupule à collaborer avec Arcand et, surtout, à le financer continuellement. En effet, comme l'explique Alfred Hardy, l'un de ses plus proches collaborateurs: «En somme, tous ceux qui ont contribué à le [Duplessis] hisser au sommet de la gloire ont été récompensés sous une forme ou sous une autre ${ }^{50}$ ".

Cette déclaration, qui interpelle directement l'ensemble des décideurs politiques de l'époque duplessiste, place ces derniers devant deux problèmes éthiques. Tout d'abord, qu'est-il permis de faire à un politicien pour rétribuer celui qui l'a aidé à prendre le pouvoir? Du point de vue d'Arcand, la survie de ses entreprises journalistiques et militantes justifie assurément tous les compromis avec les politiciens locaux, provinciaux et nationaux. Cela dit, du point de vue des hommes politiques concernés, comment peut-on justifier, en démocratie, cet appui constant et pérenne dont ont bénéficié les organisations fascistes d'Adrien Arcand? Dans les années 1930, on pouvait invoquer la méconnaissance, sinon l'ignorance des dangers réels que pouvaient représenter ces organisations fascistes. On

$$
4+4
$$

50. Alfred HARDY, Patronage et patroneux..., op. cit., p. 133. 
pouvait invoquer, également, la culture politique essentiellement électoraliste de cette période. Or, après 1945, ces éléments d'explication sont absolument insoutenables, car le monde entier connaît à ce moment-là l'existence des chambres à gaz.

Enfin, la Shoah amène les politiciens à s'interroger: est-il permis d'autoriser un individu tel qu'Adrien Arcand ou une organisation telle que le PUNC à soutenir publiquement une idéologie haineuse, qui a causé la mort de plusieurs millions de personnes en Europe? Les membres du Comité permanent des affaires étrangères de la Chambre des communes ont dû répondre à cette question lorsqu'ils se sont mis à étudier, en 1965, un projet de loi concernant le génocide et un amendement visant à interdire l'acheminement de messages haineux par la poste.

En effet, cette année-là, le fasciste John Ross Taylor dépose devant les membres du Comité un mémoire où il cite Arcand afin d'étayer ses thèses antisémites. Ce cas incite Guy Favreau, le ministre fédéral de la Justice, à mettre sur pied un comité spécial chargé d'étudier les problèmes relatifs à la diffusion de la propagande haineuse au Canada, présidé par Maxwell Cohen.

Dans son rapport, Cohen indique bel et bien qu'un très petit nombre de citoyens partagent les idées antisémites et fascistes d'Arcand. Néanmoins, suggère-t-il, les parlementaires devraient légiférer pour éviter que des gens ne diffusent de la propagande haineuse, car il n'y a, à ce moment-là, aucune loi qui interdit à un individu ou à une organisation de calomnier un groupe de personnes. Les députés suivront l'essentiel de ces recommandations lorsqu'ils amenderont, en 1970, les articles 318 à 320 du Code criminel ${ }^{51}$.

Adrien Arcand aura ainsi indirectement contribué à poser un important jalon dans notre histoire juridique : indiquer où se termine la liberté d'expression et où commence le préjugé en vue de diffamer un groupe de personnes.

$$
4+4
$$

51. Hughes THÉORÊT, Les chemises bleues. Adrien Arcand, journaliste antisémite canadien-français, Québec, Septentrion, 2012, p. 341-346. 